\title{
Design of Multi-Level Intelligent Control Systems for Complex Technical Objects on the Basis of Theoretical-Information Approach
}

\author{
Bary Ilyasov, Vladimir Vasilyev, Sagit Valeev \\ Ufa State Aviation Technical University, K. Marx Str. 12, 450000 Ufa, Russia \\ e-mail: ilyasov@tc.ugatu.ac.ru,vasilyev.vi@net.ugatu.su,valeev.ss@ net.ugatu.su
}

\begin{abstract}
The problem of designing multi-level intelligent control systems for complex technical objects on the basis of the theoretical-information approach is discussed. The proposed approach allows us to make well-grounded project decisions when designing control algorithms for advanced control systems. The problem statement of applying the proposed approach to the design of intelligent control systems for aviation gas-turbine engines is considered.
\end{abstract}

Keywords: multi-level control system; intelligent control design; entropy; aviation engine

\section{Introduction}

When designing automatic control systems (ACS) for complex technical objects some main common criteria are usually used: the global goal of control process; the final level of achieving this goal; the computational resources required to achieve the delivered goal with a given performance; the complexity and the time terms of the design process. In addition, we need to reduce the total cost of designed ACS, guarantee the reliability of the system operation under conditions of coordinate-parametric and structural disturbances action. The requirements of network interaction in the framework condition of group control and cooperation in ACS as a multiagent system are also to be kept in mind. All these problems lay in the table of designing three-aspect components of ACS (so called problem C3 Control, Computation, and Communication). Since the requirements of ACS characteristics for complex technical objects are constantly growing, the complexity of the control systems and the complexity of their design process become the key factors determining the quality of design solutions and the efficiency of ACS.

One of perspective directions in the modern control theory is the development of intelligent control systems (ICS) on the basis of IPDI (Increasing Precision with 
Decreasing Intelligence) principle [1], the essence of which consists in decomposition of control objectives and tasks depending on the requirements to precision and intelligence of control problem solution. Here, the higher is the required control precision at a hierarchical level, the lower are requirements to the control intelligence level; and on the contrary, the higher is the required level of intelligence, the lower is the necessary precision of control [2].

The application of such an approach gives a possibility to estimate and minimize the complexity of design decisions under construction of control systems for complex technical objects (CTO).

The base architecture of a hierarchical ICS consists of three vertical interacting levels: the organizational, the coordination, and the executive control level.

The first who offered the notation of control system complexity lying in the base of law of requisite variety was W. Ashby [3]. This approach was then developed by V. Solodovnikov as the principle of minimal complexity [4]. According to the given principle, the design of CTO control system is reduced to the selection of the controller structure having minimal complexity under the specified requirements to control performance.

The problem discussed below is how to formalize the procedure of designing the multi-level intelligent control system, how to set the specifications for different control levels, and how to choose the control algorithms on each of control levels having minimal structural complexity. The universal measure of the structural complexity being used here is the entropy of control processes.

The indicated tasks of designing the control systems for complex technical objects are related to the class of multicriteria optimization problems. To reduce the number of iterations during the design procedure and to ensure the required performance of control processes, it is necessary to evaluate and minimize the complexity of the project solutions at the preliminary design stage.

Let us consider below the formulation of the problem of designing the control system for the complex technical object on the example of control algorithms design for the aviation engine (AE). The prospects of such systems development are associated with the development of multi-level intelligent control systems (MICS) that ensure the fulfillment of the given set of requirements to performance, robustness, and fault tolerance of AE control processes [5].

At the same time when designing AE MICS it is necessary to take into account the rigid constraints imposed on the computational processes implementing the control algorithms [6].

The tasks of analysis and design of ACS in the class of AE MICS have a number of special features:

- the use of control algorithms based on soft computing methods (fuzzy logic, neural networks, genetic algorithms, etc.) mostly is based on the trial and error 
method. Theoretically, only the existence theorems of these algorithms are known in the literature, there are no formalized methods and techniques for analyzing and synthesizing algorithms for the intelligent control system of AE [7-17];

- it is necessary to guarantee the stability of control processes in the AE MICS both under conditions of the nominal modes and the influence of uncertainty factors (for example, in case of subsystems failures or appearance of possible structural defects in distributed databases and knowledge bases) [18].

These circumstances make it very difficult to solve the problem of design and analysis of MICS that significantly limits the use of achievements in the field of artificial intelligence theory for solving practical problems in this subject area [1920].

The paper considers the formalized approach to solving the problem of designing AE MICS based on the theoretical information approach. The proposed approach allows us to make well-founded design decisions while designing the control algorithms of advanced MICS for the new generation of AE based on the fundamental principle of minimum complexity.

\section{The Statement of the MICS Design Problem}

As an analysis shows, the conception of AE MICS design must be based on the use of the following additional system principles:

1) the principle of the functional integration which supposes a unification of different (heterogenous) subsystems;

2) the principle of the hierarchical organization, meaning the construction of the control system in a class of multi-level hierarchical control systems with a division (decomposition) into several control levels differing by a choice of control goals and implementation methods:

3) the principle of integrating the various models, methods, and algorithms of analysis and design of control system with the use of both the classical methods of multivariable control and intelligent control of complex dynamic objects;

4) the principle of the minimal complexity, supposing a choice of the simplest structure of control algorithms with the account of the change of AE operation conditions at the optimum use of information and computing resources of the control system;

5) the principle of the open system construction, being a basis of intellectualization and standardization of information processing technologies at the various stages of MICS life cycle. 
Let us suppose that any possible control situation can be defined as a triade including the control object state, the environment conditions and the control goal. The mapping $\Pi=(\pi 1, \pi 2, \pi 3)$ characterizes then a procedure of designing the control algorithms of MICS on the basis of their decomposition to three interacted control levels:

$<$ Control object $>\stackrel{\pi_{1}}{\longrightarrow}<$ Algorithms of executive level (EL) >;

$<$ Environment $>\stackrel{\pi_{2}}{\longrightarrow}<$ Algorithms of coordination level (CL) $>$;

$<$ Control goal $>\stackrel{\pi_{3}}{\longrightarrow}<$ Algorithms of organizational level (OL) $>$.

The complexity of design decisions here should correspond to the complexity of the control situation. As a measure of control situation complexity, in this case, one can use the entropy estimates:

$$
\left.\begin{array}{l}
\pi_{1}: H(Y / U, F) \text { Complexity of EL algorithms } \\
\pi_{2}: H(F) \text { Complexity of CL algorithms } \\
\pi_{3}: H(G) \text { Complexity of OL algorithms }
\end{array}\right\} \begin{gathered}
\text { Entropy as a measure of } \\
\text { design decision } \\
\text { complexity }
\end{gathered}
$$

Here $H(Y / U, F)$ is the entropy of control processes on the MICS EL-level;

$H(F)$ is the entropy of the environment conditions change;

$H(G)$ is the entropy of the control goal change;

$Y, U, F, G$ are the vectors of the measured output parameters of the control object, the control actions, the external disturbances, and the control goals respectively.

The entropy of the output parameters vector $\mathrm{Y}$ is defined in this case as

$$
H(Y / U, F)=-\int_{\Gamma_{Y}} p(Y / U, F) \ln p(Y / U, F) d Y,
$$

where $p(Y / U, F)$ is the distribution density function for the output vector $Y$. The entropy of the environment conditions change is estimated by the expression

$$
H(F)=-\int_{\Gamma_{F}} p(F) \ln p(F) d F,
$$

where $p(F)$ is the distribution density function for the vector $F$. The entropy of the control goal $H(G)$ can be estimated similarly.

With an account of the above mentioned, the problem of optimum design of threelevel MICS on the basis of entropy approach is formulated as follows.

In the general case, the description of the $\mathrm{AE}$ behavior as a multivariable dynamic control object in the state space can be represented as a system of non-linear differential and algebraic equations 
$\dot{X}=F(X, U, V)$

$Y=H(X, U, V)$

where $X \in R^{n}$ - is the state variables vector of AE; $Y \in R^{m}$ - the vector of the measured coordinates (outputs) of AE; $U \in R^{l}$ - the vector of the control actions (inputs) of $\mathrm{AE} ; V \in R^{k}$ - the vector of disturbances characterizing the external environment conditions.

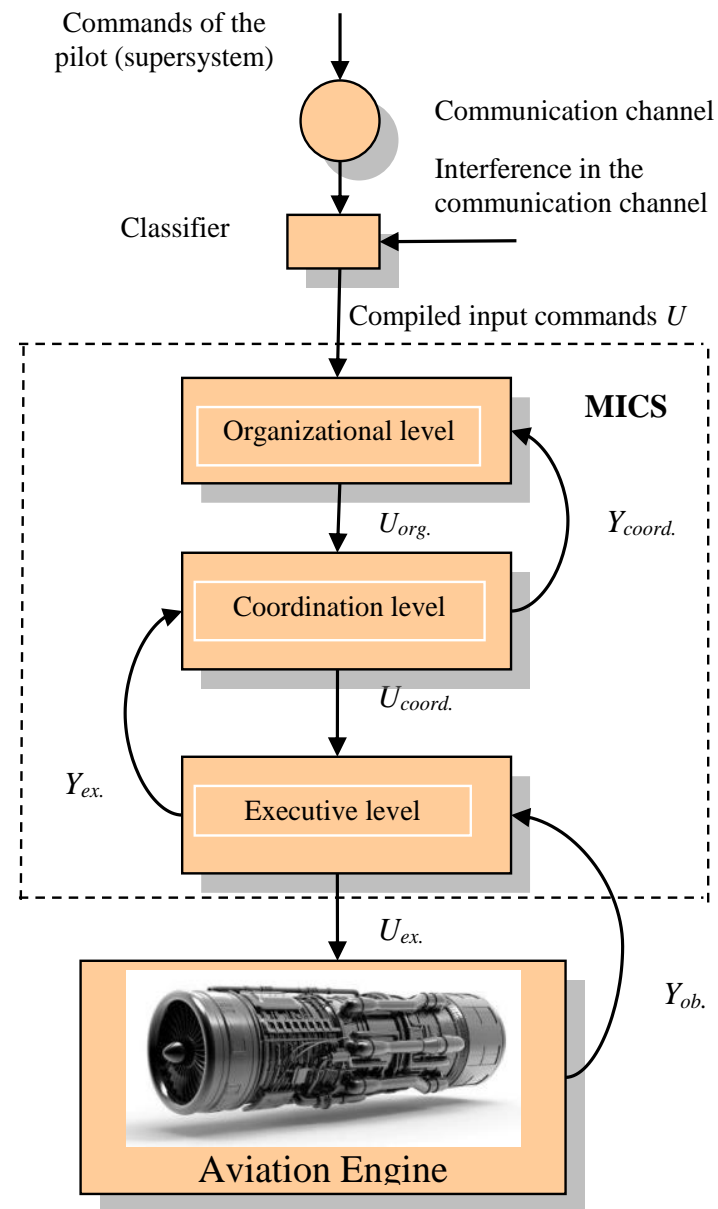

Figure 1

Multi-level control system of aviation engine

In Figure 1 the block diagram of the multi-level intelligent control system of AE consisting of the executive level, the coordination level, and the organizational level is presented. 
To achieve the settled goal of control at each level, a set of interconnected algorithms is applied, for implementation of which the following information about the current state of the control situation is used:

- $X \in \Gamma_{X}$ - the control object state vector, $\Gamma_{X}=\underset{i}{\cup} \Gamma_{X_{i}}$ - the range of the AE possible states (correspondingly regular, potentially dangerous and emergency operating modes);

- $Y \in \Gamma_{Y}$ - the vector of the state variables values available for the measurement, $\Gamma_{Y}=\cup_{i} \Gamma_{Y_{i}}$ the range of the AE values for indicated possible states;

- $U \in \Gamma_{U}$ - the control actions vector, $\Gamma_{U}$ - the area characterizing the possibility of the goal-directed change of the AE state vector;

- $F \in \Gamma_{F}$ - the vector of disturbances from the environment: $F(1)$ - the parametric disturbances; $F(2)$ - the signal disturbances; $F(3)$ - the structural disturbances (changing the configuration of the control object, failures, etc.). The range of the values for each of these disturbances classes contains, in turn, some nested regions.

Under the control goal we will understand the solution of the problem of approximation of the output parameters vector $Y$ concerning the desired result, i.e. the target vector $Y^{*}$ :

$$
\left\|Y-Y^{*}\right\| \leq \varepsilon
$$

where $\varepsilon$ is the given admissible control error; $Y^{*}$ - the desired value of the AE output variables vector at the given operating mode.

Let us assume that the optimal control reduces the entropy of the control vector concerning the desired value, i.e. ensures the following condition fulfillment:

$U_{o p t}: H_{\max }\left(Y / U_{o p t}, F\right) \leq H_{a d m}$

where $H_{a d m}$. is the admissible entropy determined by the given requirements to the control processes performance:

$$
H_{\text {adm. }}=\max _{F \in \Gamma_{F}}\left\{-\int_{\Gamma_{Y}} p_{\text {des. }}(Y) \ln p_{\text {des. }}(Y) d Y\right\},
$$

where $p_{\text {des. }}(Y)$ is the desired (given) distribution probability density, chosen on the base of specified accuracy of achieving the goal (specified for the control system in the form of the error distribution law); 
$H_{\text {max }}\left(Y / U_{\text {opt }}, F\right)=\max _{F \in \Gamma_{F}} H(Y / U, F)$ is the entropy of the output vector $Y$, corresponding to the optimal control $U_{\text {opt. }}$.

The evaluation of the desired entropy (i.e. the entropy of the target vector $Y^{*}$ ) of the ACS can be defined as follows:

$$
H\left(Y^{*}\right)=-\int_{\Gamma_{Y^{*}}} p\left(Y^{*}\right) \ln p\left(Y^{*}\right) d Y^{*}
$$

where $p\left(Y^{*}\right)$ is the distribution probability density of the vector $Y^{*}$.

The purpose of the hierarchical AE MICS design is the choice of the set of such control system algorithms that provide the specified performance of control processes under minimal complexity of control algorithms.

\section{Solution of Optimal Design Problem of AE MICS}

The following problem statement is considered: it is necessary to find such method of constructing AE MICS and its hierarchical control levels, i.e. to determine such composition of control algorithms, database structure (DB) and knowledge bases (KB) of MICS, under which the requirement (5) is fulfilled, provided that the following condition is fulfilled:

$H_{\Sigma}(A) \rightarrow \min$

where $H_{\Sigma}(A)$ is the total entropy of the set of control algorithms for all three levels of AE MICS, which can be calculated as

$$
H_{\Sigma}(A)=H_{e x .}(A)+H_{\text {coord. }}(A)+H_{\text {org. }}(A)
$$

here, $H_{\text {ex. }}(A), H_{\text {coord. }}(A), H_{\text {org. }}(A)$ are respectively the entropy (computational complexity) of the algorithms of executive-level, the coordination level and the organization level of MICS.

It is assumed that in addition to condition (5) the following restrictions must be provided also:

$$
H_{\text {max }}\left(Y, U_{o p t}, F\right) \leq H_{a d m} ; \forall X \in \Gamma_{X_{1}} ; Y \in \Gamma_{Y_{1}} ; U \in \Gamma_{U} ; \forall \mathrm{F} \in \Gamma_{\mathrm{F}},
$$

where $H_{a d m}$ is the specified (maximum admissible) entropy of MICS control processes. 
The solution of the stated design problem is carried out on the basis of the principle of sequential decomposition ("bottom-up"). In accordance with it at each of the control level, a selection of algorithms with minimal complexity satisfying the given requirements (4), (5), (8), (10) to control processes at the executive, coordination, and organization levels MICS are to be provided.

\section{Examples of AE MICS Design}

Let us consider the examples of designing the intelligent control system for the AE.

Example 1. In [6] the model of two-shaft multi-mode gas-turbine engine is presented as the set of piece-wise linear dynamic equations of the following form:

$\dot{X}=A(\eta)\left(X-X^{s s}(\eta)\right)+B(\eta)\left(U-U^{s s}(\eta)\right)$,

$Y=Y^{s s}(\eta)+C(\eta)\left(X-X^{s s}(\eta)\right)+D(\eta)\left(U-U^{s s}(\eta)\right)$.

where

$A_{i} \in R^{2 x 2}, B_{i} \in R^{2 x 2}, C_{i} \in R^{3 x 2}, D_{i} \in R^{3 x 2}$,

$X \in R^{2}, U \in R^{2}, Y \in R^{3}$;

$X=\left(n_{L}, n_{H}\right)^{T}$ is the vector of the state variables (which are defined as the AE rotation velocities of the low and high-pressure compressor rotors respectively); $U=\left(G_{f}, A_{N}\right)^{T}$ - the vector of control actions (the fuel flow rate and the jet nozzle area); $Y=\left(p_{C}^{*}, p_{T}^{*}, T_{T}^{*}\right)^{T}$ - the vector of controlled variables (the air pressure behind the compressor, the pressure and the gas temperature behind the turbine); $X_{i}^{s s}, U_{i}^{s s}, Y_{i}^{s s}$ are the values of the vectors $X, U, Y$ at the steady-state operation modes of the gas-turbine engine $(i=1 \div 4) ; \eta=\alpha \cdot n_{L}+\beta \cdot n_{H}$ - the parameter determining a selection of the $\mathrm{i}$-th point of $\mathrm{AE}$ operation mode (or respectively the i-th piece-wise linear model); $n_{L}, n_{H}$ - the relative (dimensionless) values of the variables $n_{L}$ and $n_{H}$.

If we solve the interpolation task with the use of a 3-layered neural network (NN), then the nonlinear multi-mode dynamic model of gas-turbine engine will have the following form: 


$$
\begin{aligned}
& \dot{X}=\left(\sum_{i=1}^{z} W_{i}^{1} f_{i}(X)+B^{1}\right)\left(X-X^{s s}\right)+\left(\sum_{i=1}^{z} W_{i}^{2} f_{i}(X)+B^{2}\right)\left(U-U^{s s}\right), \\
& Y=\left(\sum_{i=1}^{z} W_{i}^{3} f_{i}(X)+B^{3}\right)\left(X-X^{s s}\right)+\left(\sum_{i=1}^{z} W_{i}^{4} f_{i}(X)+B^{4}\right)\left(U-U^{s s}\right), \\
& X^{s s}=\sum_{i=1}^{z} W_{i}^{5} f_{i}(X)+B^{5}, \\
& U^{s s}=\sum_{i=1}^{z} W_{i}^{6} f_{i}(X)+B^{6},
\end{aligned}
$$

where $f_{\mathrm{i}}(\cdot)$ is the activation function of a neuron; $W_{i}^{j}$ - the tuned weights of $\mathrm{NN}$; $B_{\mathrm{j}}$ - the biases in the separate layers of $\mathrm{NN}, i=1 \ldots N,(N-$ the number of the neurons in the hidden layer).

The model (12) accuracy will depend in this case on the number of the neurons in the hidden layer. An obvious advantage of such an approach is the possibility of obtaining the required interpolation accuracy for coefficients and variables by means of NN learning.

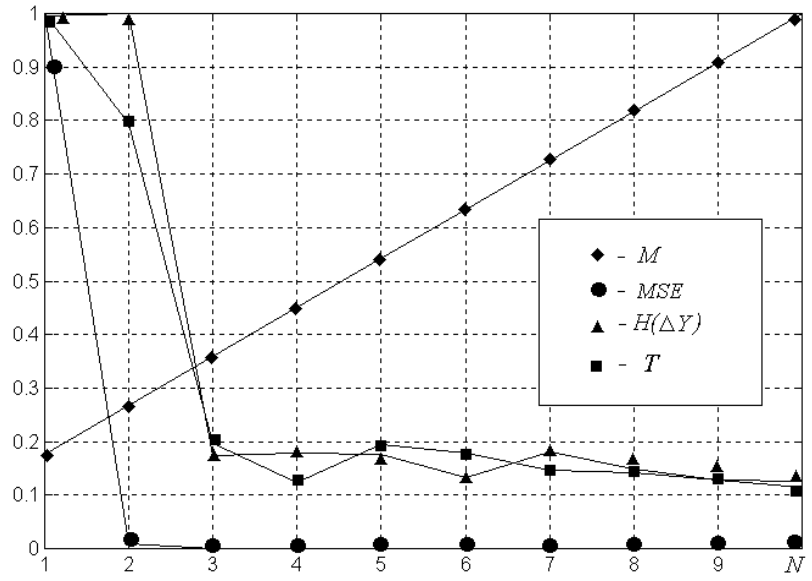

Figure 2

$\mathrm{NN}$-model characteristics depending on the number of the neurons in the hidden layer

In Figure 2 the results of $\mathrm{AE} \mathrm{NN-model} \mathrm{design} \mathrm{for} \mathrm{the} \mathrm{considered} \mathrm{case} \mathrm{are}$ presented, depending on the number of the neurons $N$ in the hidden layer (note that the scaling procedure concerning the maximal values of the variables was used). Here: MSE is the mean square error of NN learning; $T$ - the time of NN training; $M$ - the necessary volume of the memory for storage of $\mathrm{NN}$ coefficients; $H(\Delta y)$ - the entropy of NN output signals, determined with an account of (12). For estimation of $\mathrm{NN}$ algorithmic complexity it is possible to make an assumption that 
the complexity of $\mathrm{NN}$-model in our case is proportional to $M$, i.e. it grows with increasing $N$.

It follows from Figure 2 that at $N=3$ the optimum ratio between the complexity of $\mathrm{NN}$ model and its accuracy is reached.

In the process of training $\mathrm{AE} \mathrm{NN}$, there is a decrease in the entropy of the output reactions of the network due to additional information on the goals and actual learning outcomes obtained from the outside. Thus, in the process of adjusting the weights of the $\mathrm{AE} \mathrm{NN}$, it accumulates a certain amount of knowledge aimed at reducing the a priori uncertainty inherent in the beginning of the learning process.

The level of a priori uncertainty is determined in this case by the magnitude of entropy, and the ability of AE NN to assimilate the necessary knowledge is determined by the magnitude of the entropy of the weights of its interneuronal connections.

Example 2. Let us consider below the control system based on the application of the dynamic inversion method. The characteristics of the object inverse nonlinear dynamic model are realized here on the basis of NN. An inclusion of NN in the control loop provides the compensation of the object nonlinearities, facilitating a problem of forming the desired dynamic properties of the control system as a whole.

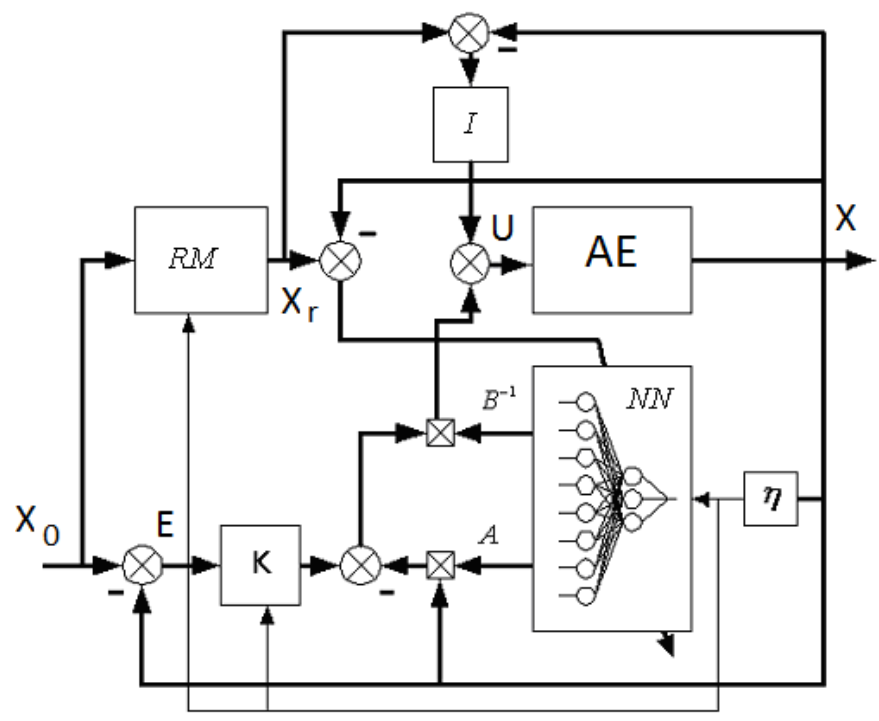

Figure 3

Gas-turbine engine control system with inverse NN-controller 
Consider the construction of a multivariable controller for $\mathrm{AE}$ as the dynamic object represented by the set of equations (12). On the basis of the approach described above, the $\mathrm{NN}$ is designed. The value of AE mode parameter $\eta$ is the input of this $\mathrm{NN}$, and at its output, the values of matrices $A(\eta)$ and $B^{-1}(\eta)$ coefficients, necessary for the compensation of nonlinearity of AE characteristics, are calculated. The performance of the transient processes in the control system is determined by the values of matrix $K$ coefficients (Fig. 3). If necessary it is possible to train again $\mathrm{NN}$ that allows us to compensate for the errors of the inverse $\mathrm{NN}$-controller and the deviations of $\mathrm{AE}$ parameters as the control object.

As the inverse model of the gas-turbine engine, the model described above can be used. It is necessary to train it for calculation of the values of matrix $B^{-1}(\eta)$.

In Figure 3 the following designations are used: $R M$ is the reference model specifying the desirable performance of the transient processes depending on $\mathrm{AE}$ operation mode $(\eta) ; I$ is the integrator, providing the zero steady-state error of the control system. The transient processes obtained for the variable $\bar{n}_{L}$ of the gasturbine engine and the variable $n_{L R}$ from the output of the reference model are presented in Figure 4.

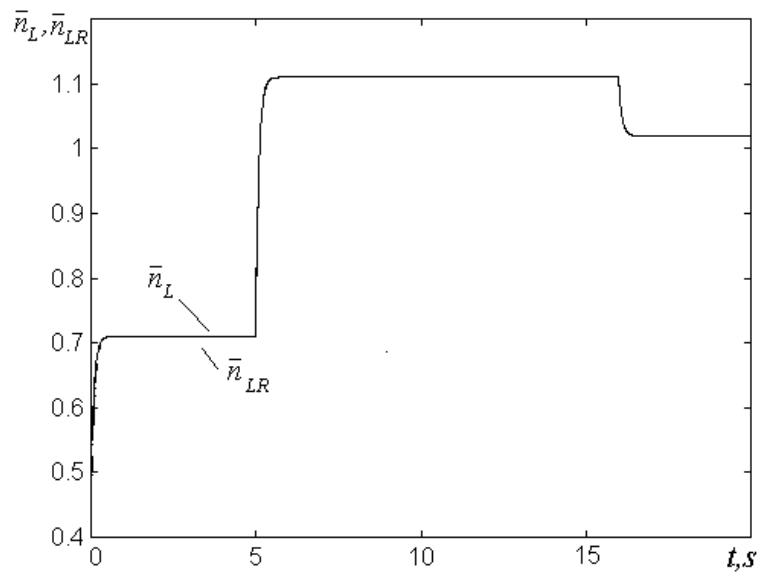

Figure 4

Simulation results of $\mathrm{AE}$ processes

As it is seen from Figure 4, the required performance of the transient processes is provided for different operation modes of the control object. 


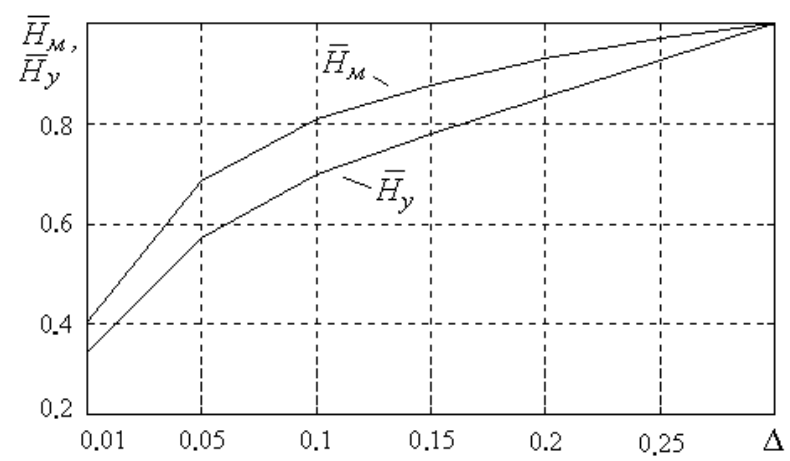

Figure 5

The change of entropy at the outputs of the system and AE inverse model

Consider the influence of the entropy at the output of the inverse NN - model on the entropy of the control system. For this purpose the numerical experiment was carried out during which the parameters of the inverse model were changed increasing a mismatch with parameters of the control object (the loop with integrator was open). Then, the entropy at the output of the inverse model was calculated as

$$
H_{M}=\log (\| \Delta A \mid * k 1)+\log \left(\| \Delta B^{-1} \mid * k 2\right)
$$

where $\Delta A\left\|, \Delta B^{-1}\right\|$ are respectively the errors of the inverse model matrices $(\cdot \|$ is a norm of a matrix), $k 1$ and $k 2$ is the scaling coefficients. In Figure 5 the curves of the entropy change at the output of AE model and the entropy at the output of the control system depending on the value of the error $\Delta$ of inverse model are presented (the plots are given for the dimensionless values:

$$
H_{M}=H_{M} /\left(H_{M}\right)_{\max }, H_{y}=H_{y} /\left(H_{y}\right)_{\max } \text {. }
$$

The entropy at the output of the control system was calculated here with the aid of the following expression

$$
H_{y}=\int_{t_{0}}^{t_{k}}\left(k_{M} \sum_{i=1}^{2} \dot{y}_{M_{i}}^{2}-k_{y} \sum_{i=1}^{2} \dot{y}_{y_{i}}^{2}\right) d t,
$$

obtained on the basis of considered in [7] approach to the entropy determination at the output of the dynamic system. As follows from Figure 5, the entropy of the control system is proportional to the entropy at the output of the inverse model. So, it is possible to set the requirements to the accuracy of gas-turbine engine inverse NN - model depending on requirements to the accuracy of the control system as a whole. For reduction of the entropy at the output of the system in this case the relearning of $\mathrm{NN}$-model is possible while the control system operation. 


\section{Conclusions}

The paper discusses the general problem of designing multi-level intelligent control systems for complex technical objects on the basis of the theoreticalinformation approach. At the same time, when designing it is necessary to take into account the rigid constraints imposed on the computational processes implementing the control algorithms. As an example, the problem of constructing the control system for an aviation engine as a complex technical object is considered. The application of the proposed approach in practice will make it possible to obtain well-grounded decisions in the field of intelligent control systems and develop high-effective multi-level control algorithms for advanced technical objects.

\section{References}

[1] Saridis G. N. Hierarchically Intelligent Machines, World Scientific Pub., Singapore, 2001

[2] Saridis G. N. Entropy in Control Engineering, World Scientific Pub., Singapore, 2001

[3] Ashby W. R. Introduction to Cybernetics, Stanford, 1955

[4] Solodovnikov V. V., Biryukov V. F., Tumarkin V. I. Principle of Complexity in Control Theory, Moscow, Nauka Pub., 1977 (In Russian)

[5] Problems of Design and Development of Gas-Turbine Engine Control Systems / S. Kusimov, B. Ilyasov, V. Vasilyev, S. Valeyev, et al. Moscow, Mashinostroyenie Pub., 1999 (In Russian)

[6] Neurocomputers in aviation (aircrafts) / Eds.: V. Vasilyev, B. Ilyasov, S. Kusimov, Moscow, Radiotechnica Pub., 2004 (In Russian)

[7] Yu H. Network Complexity Analysis of Multilayer Feed-forward Artificial Neural Networks. - In: Schumann J., Lin Y. (Eds.), Applications of Neural Networks in High Assurance Systems, SCI 268, Springer - Verlag, Berlin, Heidelberg, 2010, pp. 41-55

[8] Rzevski G. A. Practical Methodology for Managing Complexity // Emergence: Complexity \& Organization // International Transdisciplinary Journal of Complex Social Systems. 2011, Vol. 13, No. 1-2, pp. 38-56

[9] Funahashi K. On the approximate realization of continuous mappings by neural networks // Neural Networks. 1989, Vol. 2, pp. 183-192

[10] Hornik K. M., Stinchombe M., White H. Multilayer feedforward networks are universal approximators // Neural Networks. 1989, Vol. 2, pp. 359-366

[11] Pinkus A. Approximation theory of the MLP model in neural networks // Acta Numerrica. 1999, Vol. 8, pp. 143-195

[12] Gomez I., Franco L., Jerez J. Neural Network Architecture Selection: Can Function Complexity Help? / Neural Pro-cess Lett., 2009, 30, pp. 71-87 
[13] Kon M. A., Plaskota L. Complexity of Predictive Neural Networks. In: Unifying Themes in Complex Systems. Chapter 18, 2006, Springer Verlag, pp. 181-191

[14] Silva L., J. M. de Sa, Alexandre L. A. Neural network classification using Shannon's entropy // Proc. of the $13^{\text {th }}$ European Symposium on Artificial Neural Networks, Bruges, Belgium, April 27-29, 2005

[15] Bianchini M., Scarselli F. On the Complexity of Neural Network Classifiers: A Comparison Between Shallow and Deep Architectures // IEEE Transactions on Neural Networks and Learning Systems. August 2014, Vol. 25, No. 8, pp. 1553-1565

[16] Kurkova V. Complexity of Shallow Networks Repre-senting Finite Mapping. In: Artificial Intelligence and Soft Computing // Proceedings of the $14^{\text {th }}$ International Confer-ence ICAISC, 2015, pp. 39-48

[17] Erdogmus D., Principe J. C. Entropy minimization algorithm for multilayer perceptrons // Proceedings of International Joint Conference on Neural Networks (IJCNN'01) 2001, Vol. 4, pp. 3003-3008

[18] Vasilyev V., Valeyev S., Sun Jianguo, Identification of Complex Technical Objects on the Basis of Neural Network Models and Entropy Approach // Proc. of the $9^{\text {th }}$ World Multi-Conference on Systemics, Cybernetics and Informatics, Vol. IX, July 10-13, 2005, Orlando, USA, 2005, pp. 89-93

[19] Advanced Multivariable Control Systems of Aeroengines / Eds.: Sun Jianguo, V. Vasilyev, B. Ilyasov, Beijing, China, 2005

[20] Vasilyev V. I., Valeyev, S. S., Sun J. Identification of Complex Technical Objects on the Basis of Neural Network Models and Entropy Approach, Proc. of the WMSCI 2005 - The $9^{\text {th }}$ World Multi-Conference on Systemics, Cybernetics and Informatics, Vol. 9, pp. 89-93, 2005 